Pacific Northwest

National Laboratory

Operated by Battelle for the

U.S. Department of Energy

\title{
3D-FFT for Signature Detection in LWIR Images
}

\author{
PA Medvick LL Nuffer \\ MA Lind HP Foote \\ PS Mackey
}

November 2007

Prepared for the U.S. Department of Energy

under Contract DE-AC05-76RL01830 


\title{
DISCLAIMER
}

This report was prepared as an account of work sponsored by an agency of the United States Government. Neither the United States Government nor any agency thereof, nor Battelle Memorial Institute, nor any of their employees, makes any warranty, express or implied, or assumes any legal liability or responsibility for the accuracy, completeness, or usefulness of any information, apparatus, product, or process disclosed, or represents that its use would not infringe privately owned rights. Reference herein to any specific commercial product, process, or service by trade name, trademark, manufacturer, or otherwise does not necessarily constitute or imply its endorsement, recommendation, or favoring by the United States Government or any agency thereof, or Battelle Memorial Institute. The views and opinions of authors expressed herein do not necessarily state or reflect those of the United States Government or any agency thereof.

\author{
PACIFIC NORTHWEST NATIONAL LABORATORY \\ operated by \\ BATTELLE \\ for the \\ UNITED STATES DEPARTMENT OF ENERGY \\ under Contract DE-AC05-76RL01830
}

Printed in the United States of America
Available to DOE and DOE contractors from the Office of Scientific and Technical Information,
P.O. Box 62, Oak Ridge, TN 37831-0062;
ph: (865) 576-8401
fax: $(865)$ 576-5728
email: reports@adonis.osti.gov

\begin{abstract}
Available to the public from the National Technical Information Service, U.S. Department of Commerce, 5285 Port Royal Rd., Springfield, VA 22161 ph: (800) 553-6847 fax: $(703) 605-6900$ email: orders@ntis.fedworld.gov online ordering: http://www.ntis.gov/ordering.htm
\end{abstract}

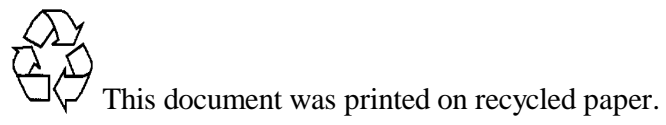




\title{
3DFFT for Signature Detection in LWIR Images
}

by

Patricia A. Medvick, Lisa L. Nuffer, Patrick S. Mackey, and Harlan P. Foote

\begin{abstract}
Introduction

A vast amount of hyperspectral data is being collected for remote sensing applications. One of the many uses for this data is locating, identifying, and quantifying chemical plumes for evaluating environmental contributions at manufacturing sites. The analysis goal is finding pixels containing a gas with a given spectral signature in a hyperspectral image (HSI) data cube. A review and comparison of methods is provided by Young (2002). The 3 Dimensional Fast Fourier Transform Matched Filter (3DFFTMF), is a variant of the whitened matched filter (WMF) which, in general, finds a known pattern in a dataset with correlated noise. This correlated noise, uninteresting variation in the data that occurs in multiple spectral bands, is evident in Fig. 1. As is typical, these spectral bands (layers) are highly correlated, contain correlated clutter.
\end{abstract}
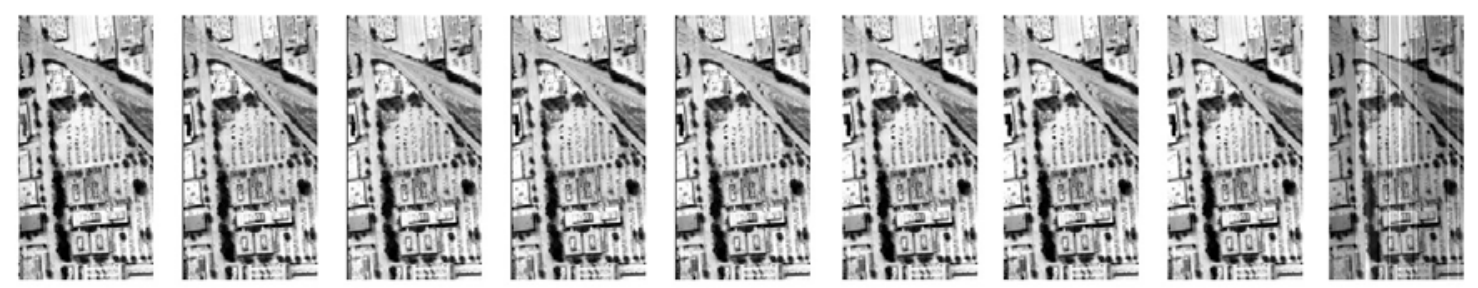

Fig. 1. Sample spectral layers of a hyperspectral datacube portray a remotely-sensed image. These images represent bands 1, 17, 33, 49, 65, 81, 97, 113, and 128 in an urban date cube composed of 128 bands. They demonstrate the high degree of correlation among spectral bands that whitening can suppress.

The WMF can be applied in the spatial or spectral domain, and uses a simplified data model that portrays the image as consisting of the signal and correlated clutter. Various whitening techniques can optimize clutter suppression (Medvick, et al. 2007) to decorrelate the noise. Once the noise is decorrelated, a filter to maximize the signal of the spectral signature of interest is constructed using a library signature of that spectrum. An example spectral signature vector of a gas is displayed in Fig. 2. Note that the spectral range encompasses the Infra Red which means that temperature will play a role in analyses. Applying the filter to the whitened image brightens the output image pixels containing the signature of interest. The brightening of the plume results in white pixels when the plume is warmer than the background, and in black pixels when the plume is cooler than the background. For consistency and easier viewing we chose the convention of displaying the brightening in the images as whitening, i.e. all plumes are white. 


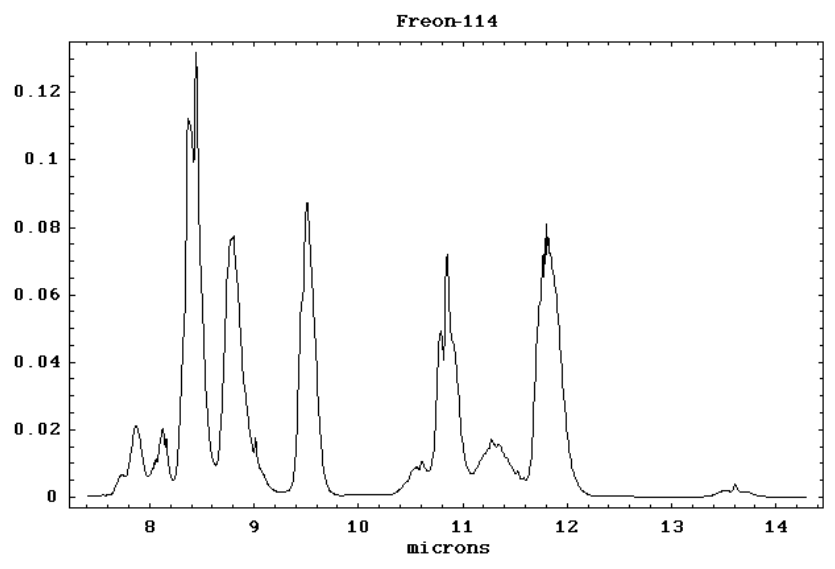

Fig. 2. An example gas signature as defined in a laboratory.

Specific versions of the WMF have been developed within the Department of Energy (DOE) community (e.g., Gas Tool Kit (GTK) at Lawrence Livermore National Laboratory, and HSI Image Processing (HIP) at Los Alamos National Laboratory). These standard spectral demixing techniques model the HSI datacube as a background illumination of a "thin" gas plume which reduces specific spectral illumination in bands of volumetric spatial absorption plume regions. These are then projection-mapped to a specific imaged "plume" pixel of the sensor spectral measurement band. The techniques detect specific signatures using a WMF composed of two parts:

1) a library of spectral representations of the expected chemical signatures -1 dimensional (1D) as a plume-pixel signature template, and

2) a second order (i.e., Gaussian) representation of the statistical clutter, as a spectral whitening, inverse-Covariance function (e.g., 1D).

The analysis tool kits use isolation of the image region of interest (ROI) containing the detected plume to estimate the chemical species and burden (product of chemical concentration and optical path length) of the plume. This WMF approach is optimum for signature detection in "colored" Gaussian noise, because it maximizes the signal-to-noise ratio (SNR) at each spectral line at a constant false alarm rate. In a sense, the WMF only detects spectral signature lines where there is no clutter, which reduces the false alarm probability. Bright pixels in the 1D WMF-output are considered plume containing pixels, and, with a known ground truth of actual plume locations in the data, the spatial count of bright WMF pixels are related to the plume detection probability.

Our work extends these traditional techniques of matched filtering signature detection by incorporating spatial as well as spectral information. Instead of a single-dimensional matched filter correlated separately along each pixel of the hyperspectral data cube (i.e. pixel by pixel), a 3D filter cube is correlated with the entire cube. This technique increases the signal to noise ratio in the filtered image by taking advantage of the fact that plume pixels containing the signature of interest are localized near one another. For greater processing efficiency and because of a potential for improved whitening techniques, the matched filtering is performed in the Fourier domain. This report 
describes, evaluates, and demonstrates application of the 3DFFTMF to the chemicalplume detection problem.

Theoretically the 3DFFTMF has an advantage over the WMF since

1) it can combine spatial and spectral filters in one application, and

2) the spatial part can be a correlation, not just a filter or smoothing, but actually looking

for a spectral pattern and spatial pattern at the same time, e.g. looking for a triangle with a specific spectrum.

In our current implementation of 3DFFTWF a filter for smoothing/averaging uses a spatial approach within the Fourier domain. If we were to look for a specific plume shape, a plume at a different angle would be ignored.

In this report we will describe our implementation of the 3DFFTMF and compare the results from this matched filter and more standard versions.

\section{DFFT implementation}

For applying a 3D analysis of hyper/multispectral data the spectral layers are treated as the $3^{\text {rd }}$ dimension with the spatial dimensions as the other 2 dimensions. The logical diagram (Fig. 3) displays in detail the steps of applying the 3DFFTMF to a data cube. The inputs are

1) the 3D datacube that may contain a region with the spectrum of interest and

2) a 3D filter representation of the spectrum of interest constructed as described below (Fig. 4).

Both inputs are subjected to the 3DFFT which shifts them into frequency rather than spatial space. Whitening in the Fourier space is achieved by creating a smoother from the Fourier version of the datacube to apply to the Fourier-version of the spectral filter cube, creating a Whitened 3D Matched Filter (W3DMF). Multiplying this W3DMF by the Fourier version of the datacube is equivalent to correlation in the spatial domain; thus applying the 3D inverse FFT to the result produces the filtered image datacube in which one layer contains the brightened pixels that correspond to the signature of interest, if present. 


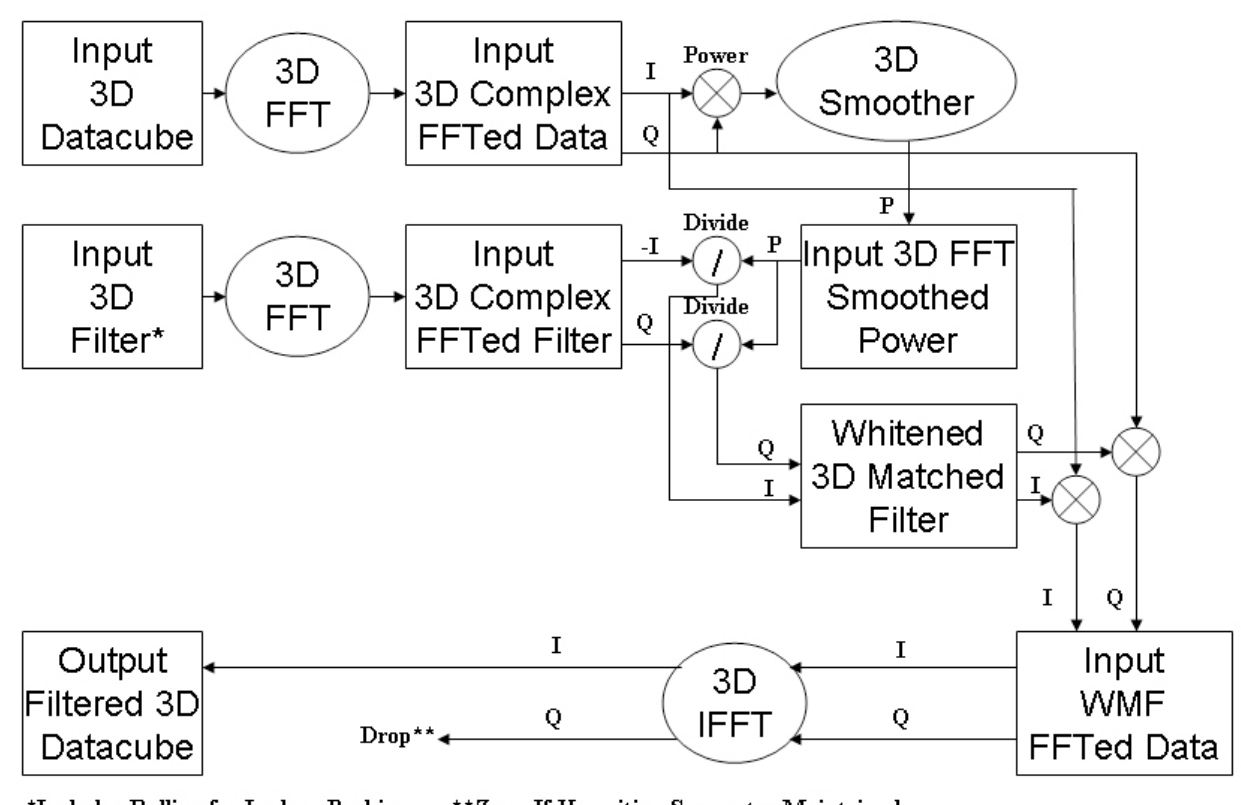

Fig. 3. Overview of steps for using the 3DFFT as a Whitened 3D Matched Filter on a 3D datacube.

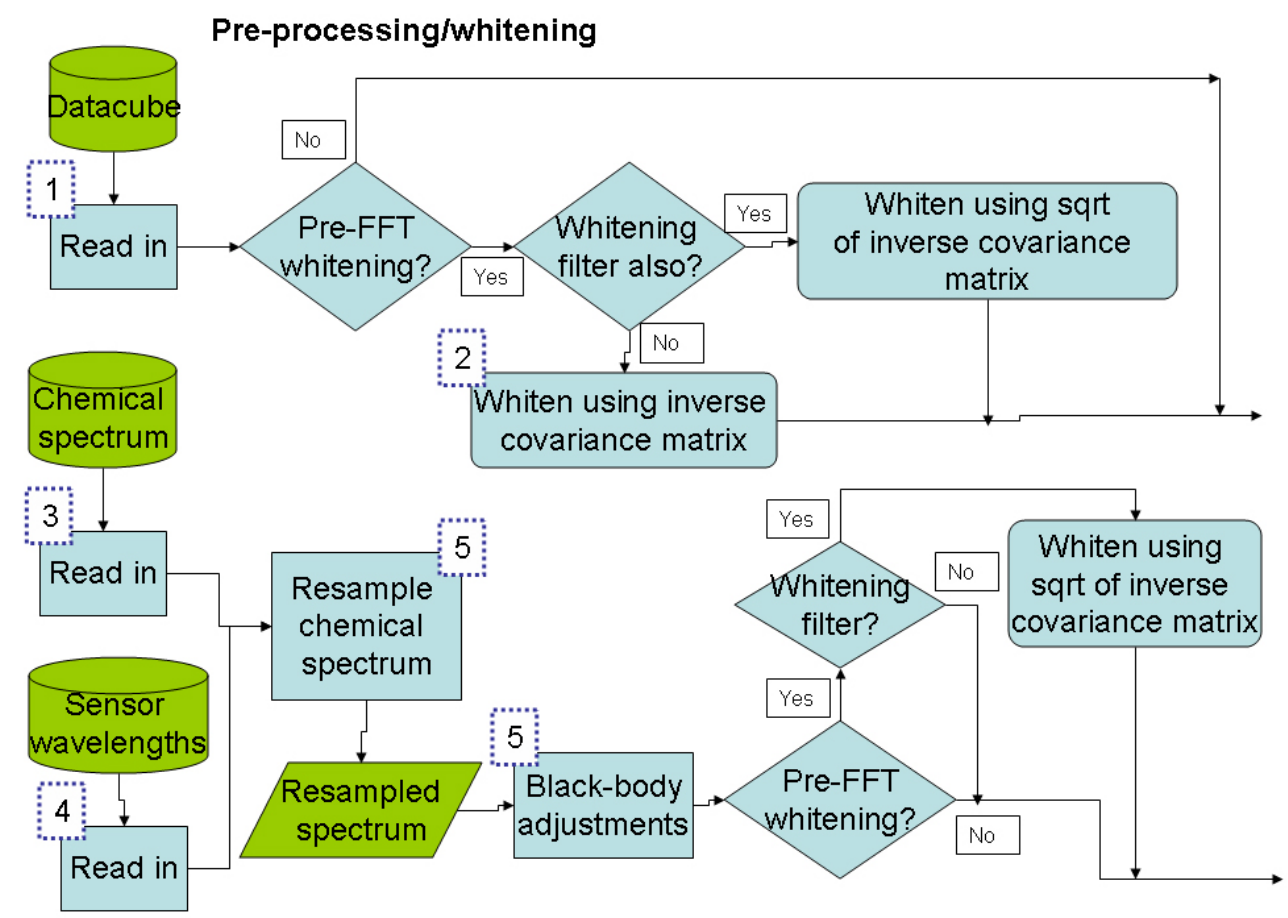

Fig. 4. Block diagram of processing steps for implementation of pre-processing steps for 3DFFTMF. 
The detailed steps in pre-processing/whitening are displayed in Fig. 4. For our initial work, whitening techniques were standardized to facilitate isolating performance differences among the matched filtering techniques. The data inputs are the 3D datacube, a chemical spectrum, and the wavelengths of the sensor. There is an option for whitening the datacube to reduce the noise. Since

1) the filter (information about the signal of interest: chemical spectrum) and datacube processed results are multiplied together (step 9- Fig. 5),

2) both the filter and the datacube may be whitened, and

3) the whitening step is multiplying by the square root of the inverse covariance matrix (ICM) of the datacube,

pre-FFT whitening may be accomplished either by multiplying both the datacube and filter by the square root of the ICM or by multiplying the data cube by the ICM.

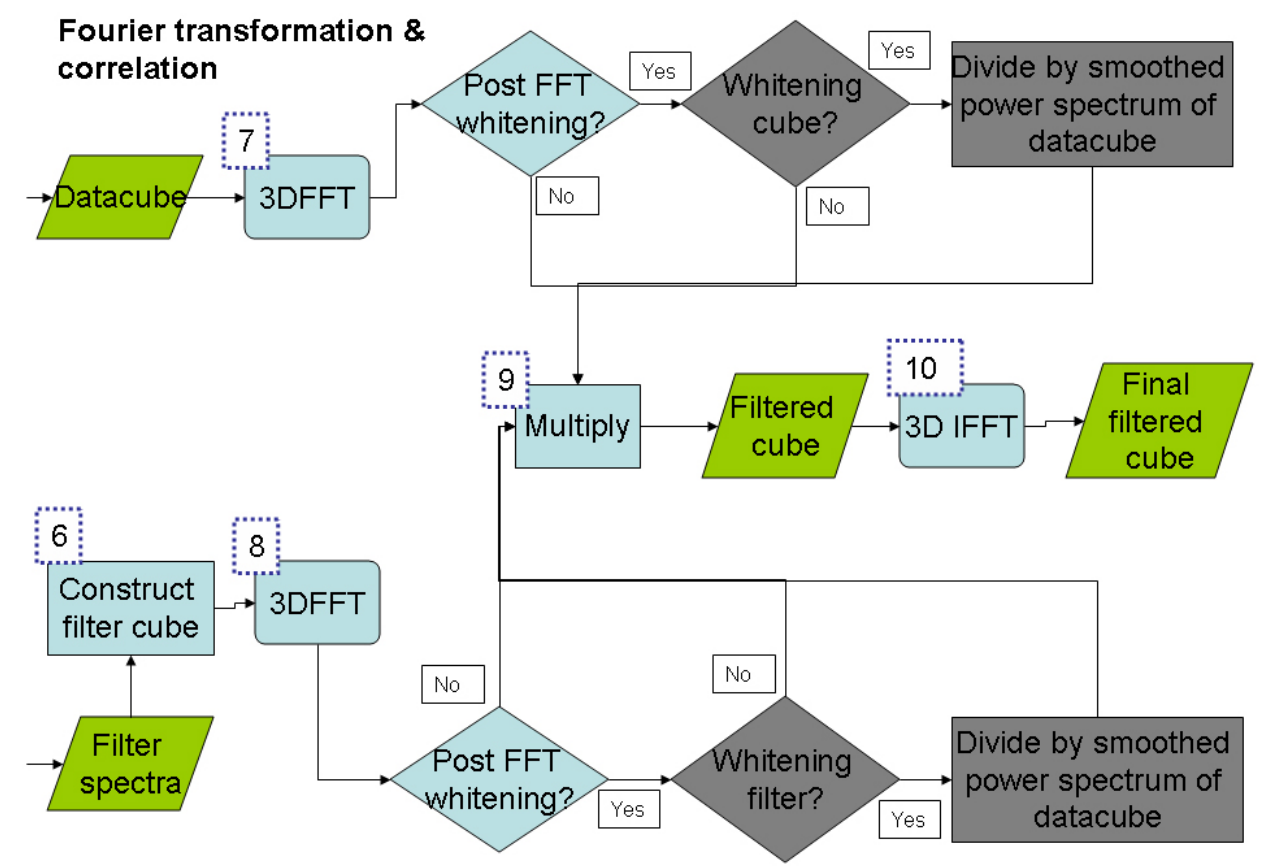

Fig. 5. The Fourier transform and correlation of the datacube and spectral inputs.

The 3DFFTMF requires information about the object signature. With hyperspectral images the spectral signature of the object in the image format of the camera sensors is achieved by sampling a finely delineated spectrum of the object in a manner that matches the sensor pattern of spectrum sampling. Using environmental information to adjust for differences between sampling in a natural environment and the standards spectra measurements creates the necessary comparison spectra. The resulting one dimensional spectra must be expand by replication into a cube to match the spatial size of the hyperspectral datacube.

Both the 3D datacube and filter cube are subjected to a 3DFFT. Each of the results may be whitened by the smoothed power spectrum of the datacube. The 2 cubes (whitened again or not) are then multiplied together, and the resulting filtered cube subjected to an inverse 3DFFT. The resulting cube tends to contain the signature of interest in one band. 


\section{Results}

\section{D Example of FFTMF}

The following example demonstrates the effectiveness of using an FFT matched filter (FFTMF) for detecting spatial objects in a two-dimensional image. Distinctive light pole features (Fig. 6) were used as signatures to create a matched filter. The filter was correlated with the whitened image using two WMF techniques. Traditional matched filtering simply correlates the matched filter with the whitened image. The FFTMF performs the mathematically equivalent operation in the Fourier domain by performing a Fourier transform on both the filter and the whitened image, multiplying them together, and performing an inverse transform on the result.

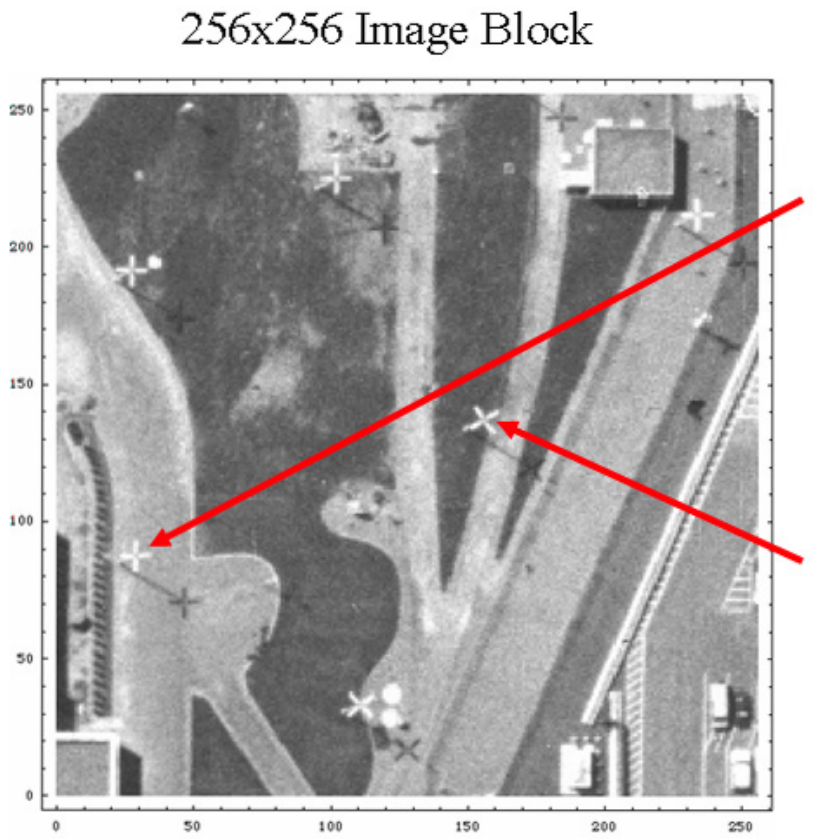

\section{Lamp post}

$17 \times 17$ Pixel Signature

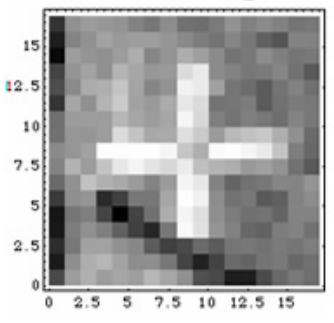

\section{Angled Signature}

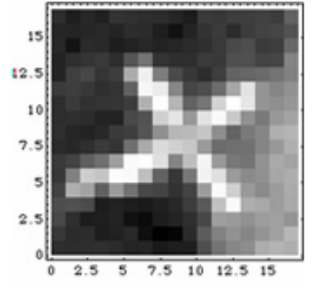

Fig. 6. Image and light pole signatures used for 2D example of FFTMF spatial detection

The results from the traditional WMF using a spatial signature are shown in Figs. 7 and 8. Each representative 17 by 17 pixel patch containing the light pole is transformed into a matched filter. Correlation with the whitened image results in a white (brightened) dot at the location of a signature match. A signature match with a shadow of a light pole results in a blackened dot. The figures show the success with the horizontal and diagonal signatures as evident in the filtered images which contain 4 white dots in Fig. 7 and 2 white dots in Fig. 8. 


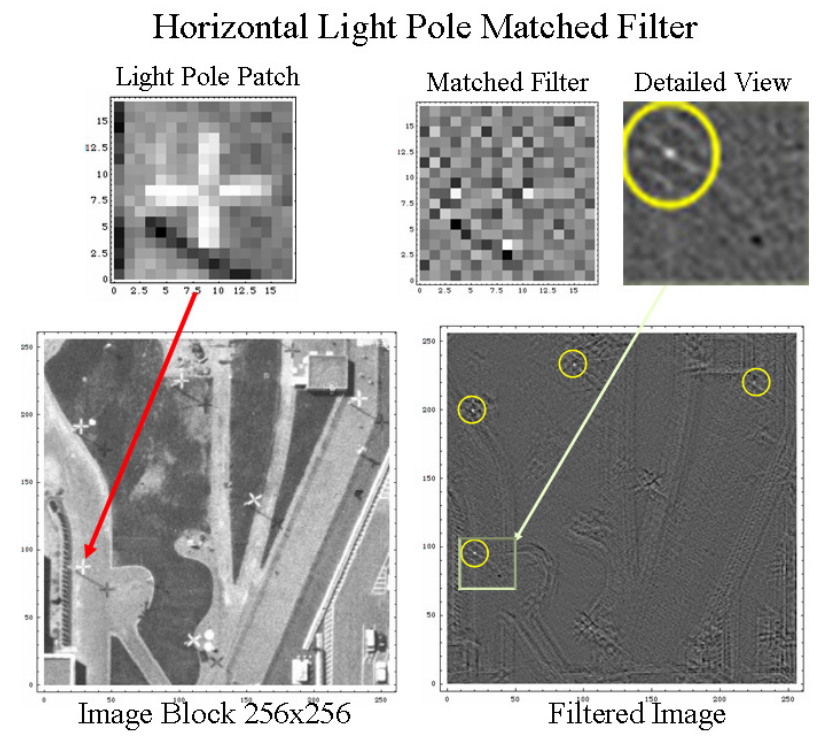

Fig. 7. Spatial matched filter results for horizontal light poles. The white dots (brightened pixels) in the filtered image indicate a successful match. The black dot evident in the detailed view is a match with the shadow of the light pole.

Diagonal Light Pole Matched Filter

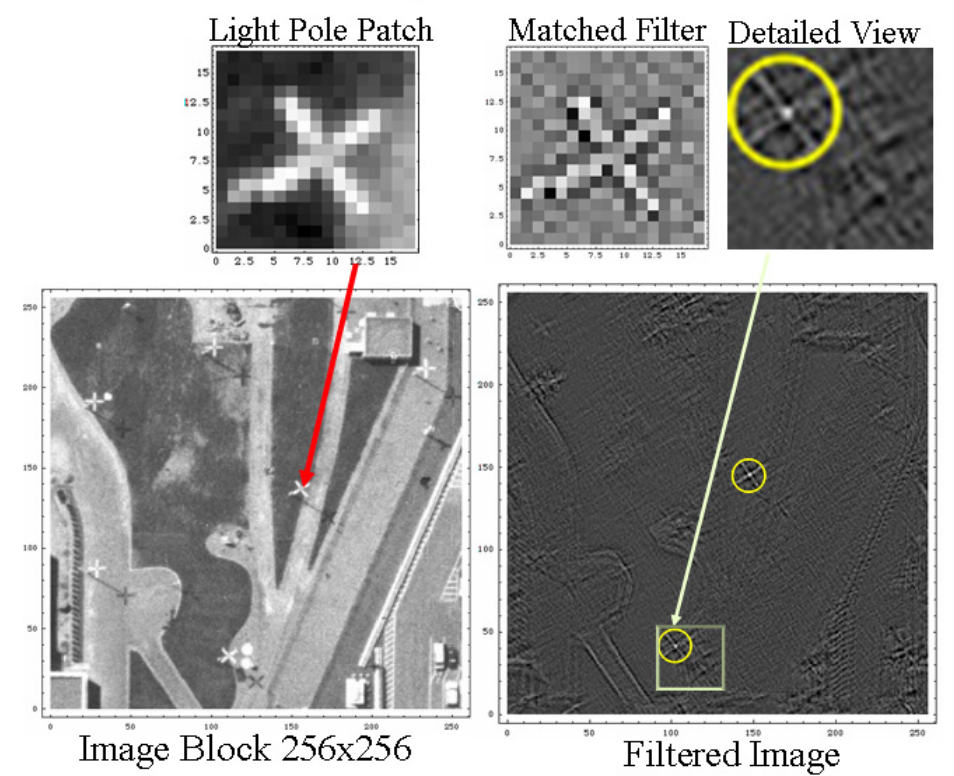

Fig. 8. Spatial matched filter results for angled light pole. The 2 white spots indicate successful spatial pattern matches. The largest black dot evident in the detailed view is a match with the shadow of the light pole.

To create a fair comparison with the spatial WMF results the image was whitened before applying the FFTMF. Fig. 9 shows the reduction in clutter that results from applying the power spectrum. The smoothed power spectrum was more successful at reducing the noise in the image. The 2D FFTMF applied the horizontal light pole signature to the whitened image. Successful detection is demonstrated by the 4 white dots evident in the resulting output image (Fig. 10). 


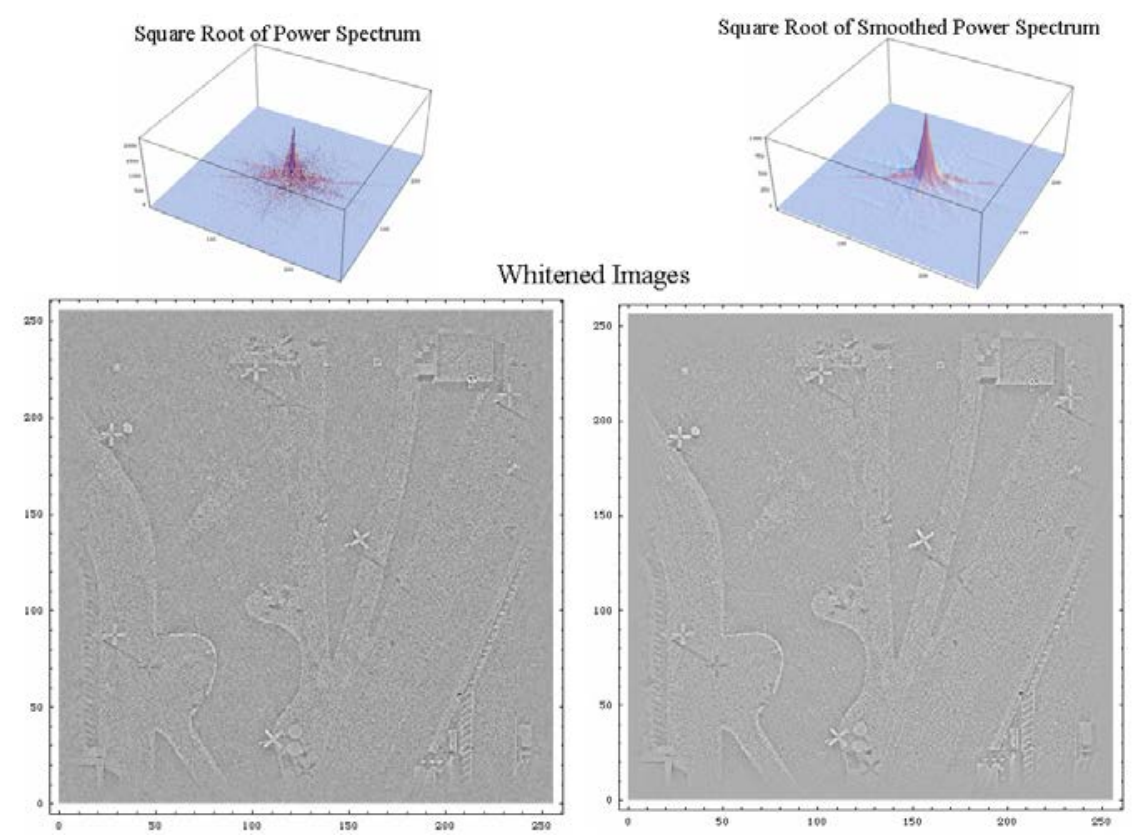

Fig. 9. Results from whitening the input image. The left image is the result of applying the power spectrum while the right image was whitened using the smoothed power spectrum. Notice the greater reduction in image clutter in the right image.

\section{D Fourier Matched Filter}

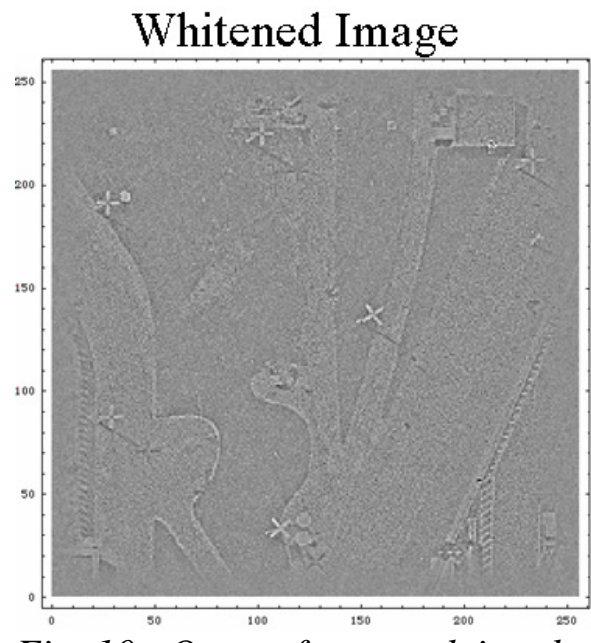

Light Pole Patch Matched Filter Output
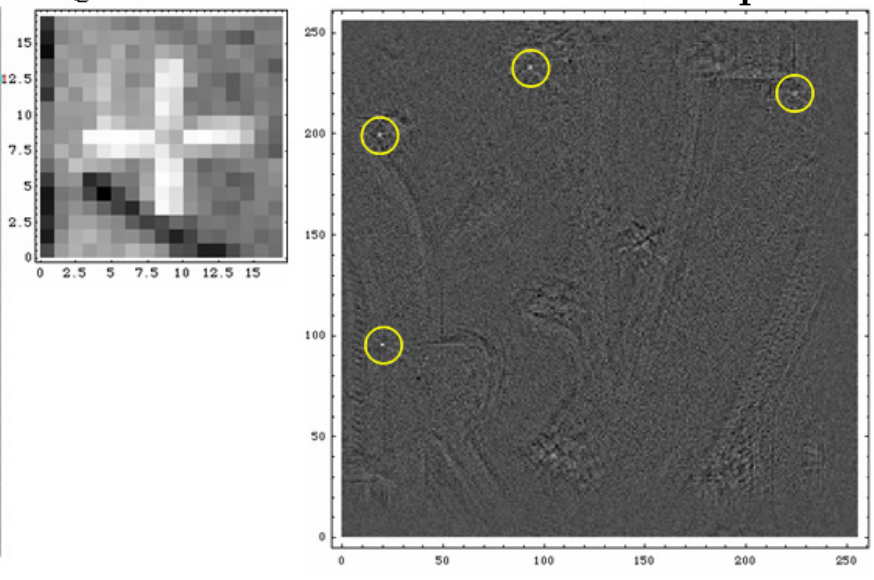

Fig. 10. Output from applying the $2 D$ Fourier matched filter to the whitened image. The yellow circles mark the 4 white dots that correspond to the horizontal light poles.

The lamp post detections were consistent between the Fourier-domain and traditional spatial-domain matched filtering techniques (Fig. 11). Thus, both versions of the WMF can use spatial patterns for matching. 


\section{D Matched Filter Comparison: Spatial}
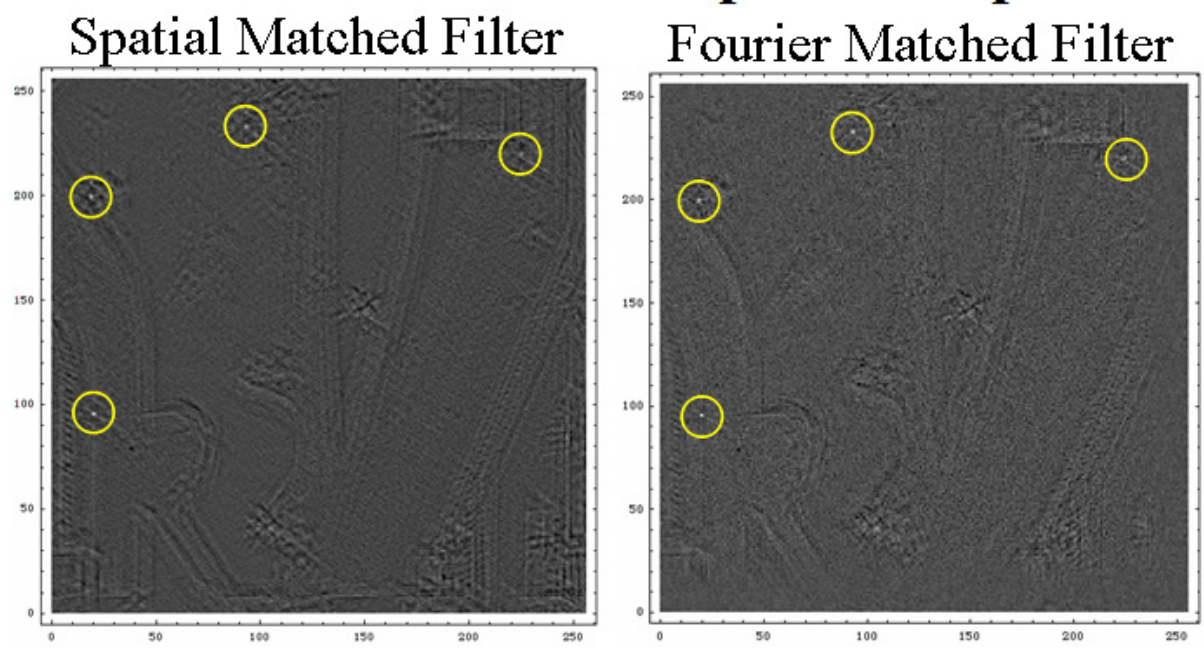

Fig. 11. The results from the spatial matched filter and the Fourier matched filter. The white dots represent successful matches.

\section{Hyperspectral datacube analyses - Desert scenerio}

In comparisons of $3 \mathrm{WMF}$ techniques on a gaseous plume in a desert scenario, PNNL's implementation, HIP with default settings, and 3DFFTWF (Fig. 12) demonstrated the success of the 3DFFT version. The $\mathrm{S} / \mathrm{N}$ ranged from $16.5 \mathrm{db}$ from the PNNL WMF to $18.22 \mathrm{db}$ for the default HIP WMF output with the 3DFFTMF between them at $17.1 \mathrm{db}$. Applying a $2 \times 2$ spatial filter to the output images improved the $\mathrm{S} / \mathrm{N}$ to 19.8 for the PNNL WMF and $20.5 \mathrm{db}$ for the 3DFFTMF (Fig. 13). Further post-WMF spatial filtering is explored in the following section.

\section{WMF Output Comparison (Desert)}
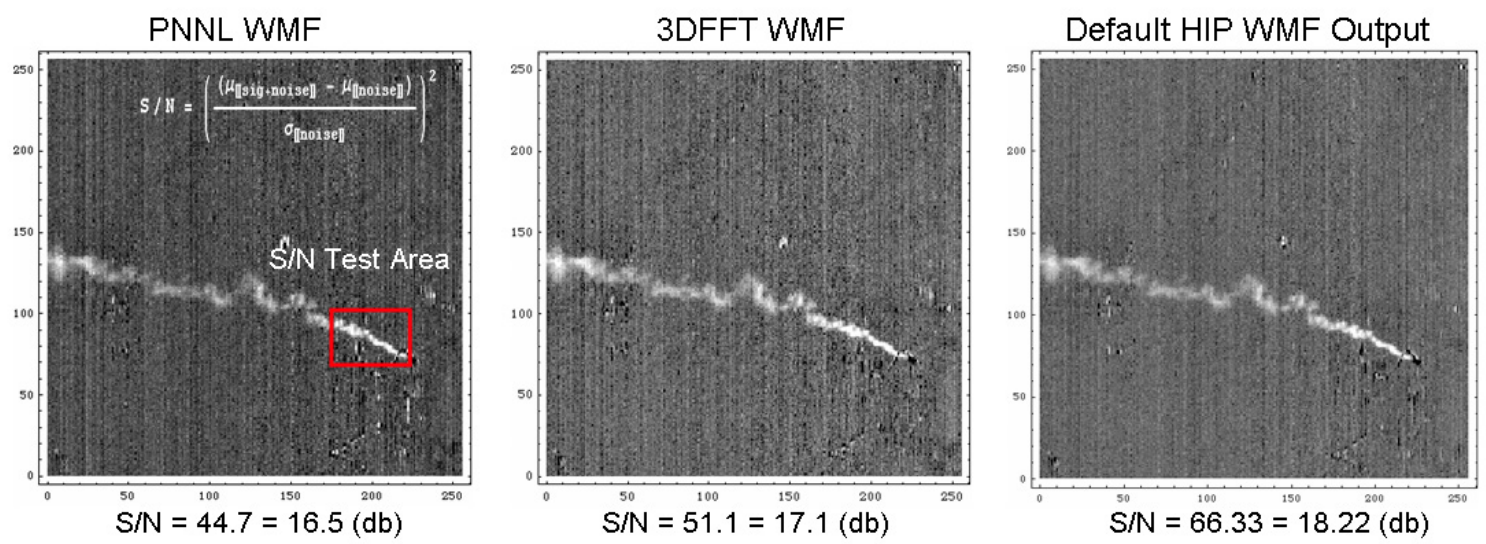

Fig. 12. Results on a data cube, whitened with full cube whitening, and subjected to 3 versions of the WMF. The same region in each of the images was the basis for the $S / N$ calculations. 


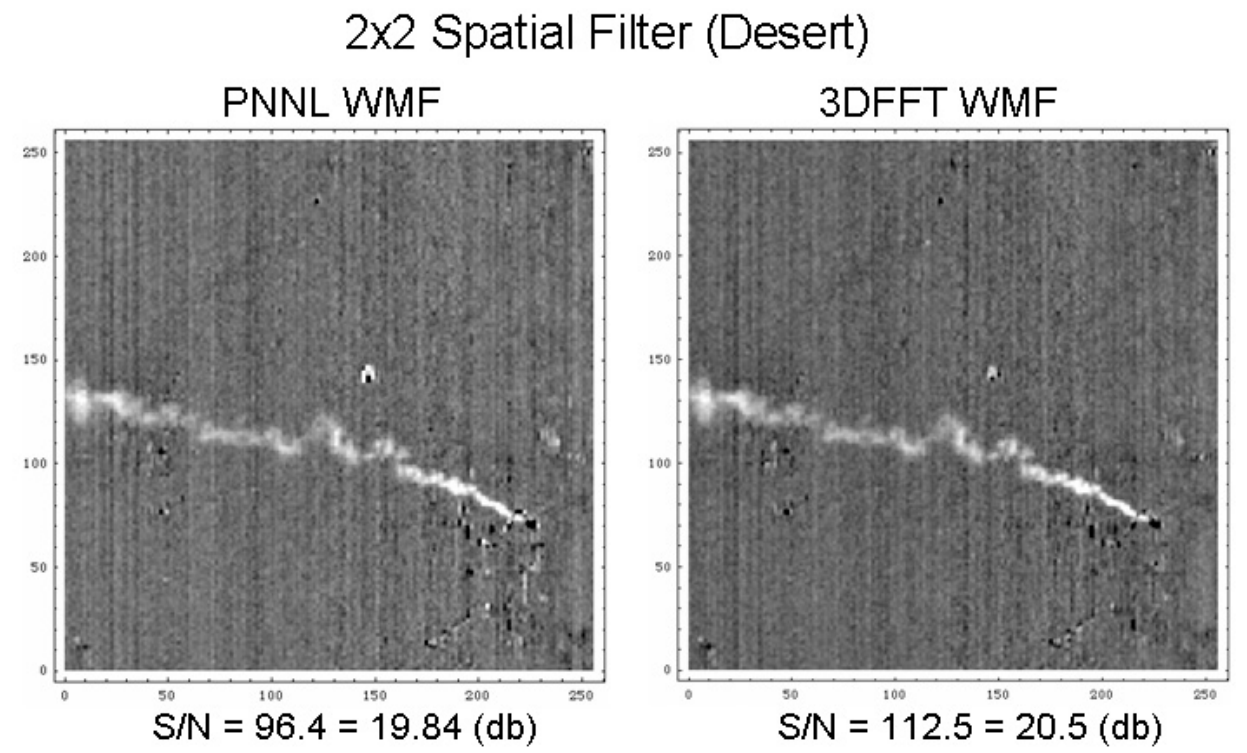

Fig. 13. Results of applying a $2 \times 2$ spatial filter to the PNNL WMF and 3DFFTWF output shown in Fig. 12. The S/Ns were calculated with the same plume region and formula as the previous figure.

\section{Spatial Post-Filter}

The FFTMF can be extended into three dimensions for hyperspectral datacubes, making it possible to match an object spectrally and spatially simultaneously. Along the spectral dimension, the filter consists of the matched spectra equivalent to that used in a 1D spectral matched filter. Spatially the filter is designed to match the object of interest, useful for objects of consistent spatial patterns. An application of the WMF can only match in one of these dimensions.

In our current implementation of the 3DFFTWF we included a spatial filtering capability after the spectral filtering and within the Fourier domain. The filter shape is circular with consistent intensity and is applied in a manner that results in low pass filtering. An inherent tradeoff in this filtering is that in the attempt to reduce noise the signal may also be reduced if the filter is larger that what is being hunted. A range of filter sizes was tested to clarify the relationship to the size of the plume. An example analysis in a desert scene produced maximum $\mathrm{S} / \mathrm{N}$ when the spatial filter size was the same as the diameter of the plume (Fig. 14). The S/N values peaked at the diameter of the spatial filter that corresponds to the plume width at the zone of testing (Figs. 15 and 16). Thus, the optimal filter size corresponds to the size of the portion of the plume of most interest. Blindly selecting a filter size could result in erasing the plume. 


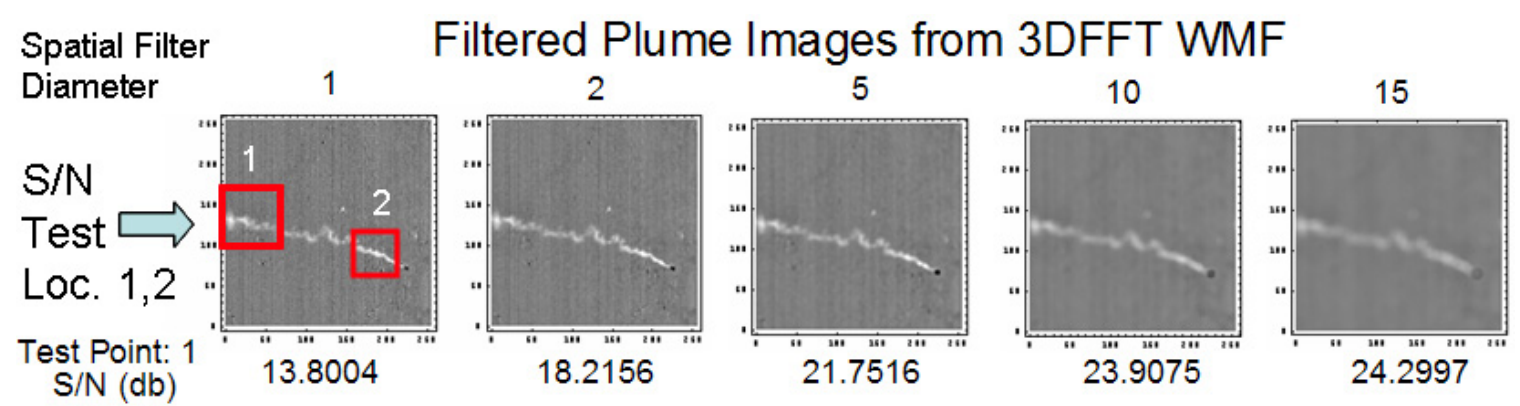

Fig. 14. Selected images from applying the 3DFFT WMF with spatial filter diameters (120) in a datacube from a desert scene. The best $S / N$ for test location 1 occurred at a filter size of 15 pixels, the diameter of the plume in test location 1. These results are graphed in Fig.15.

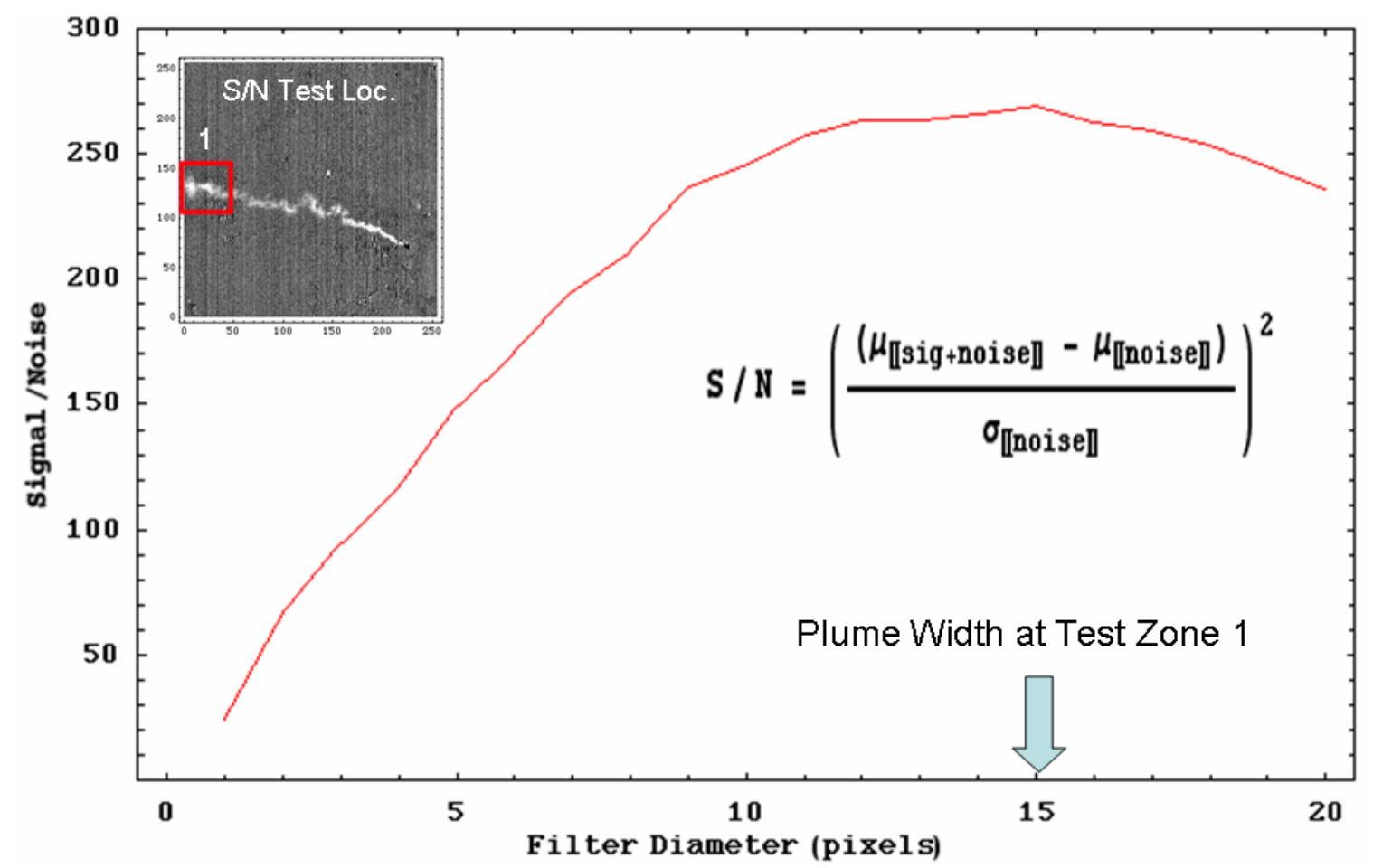

Fig. 15. S/N at test case 1 as a function of spatial filter diameter. In this desert scene the $S / N$ peaked when the filter diameter equaled the plume width in the region of interest. 


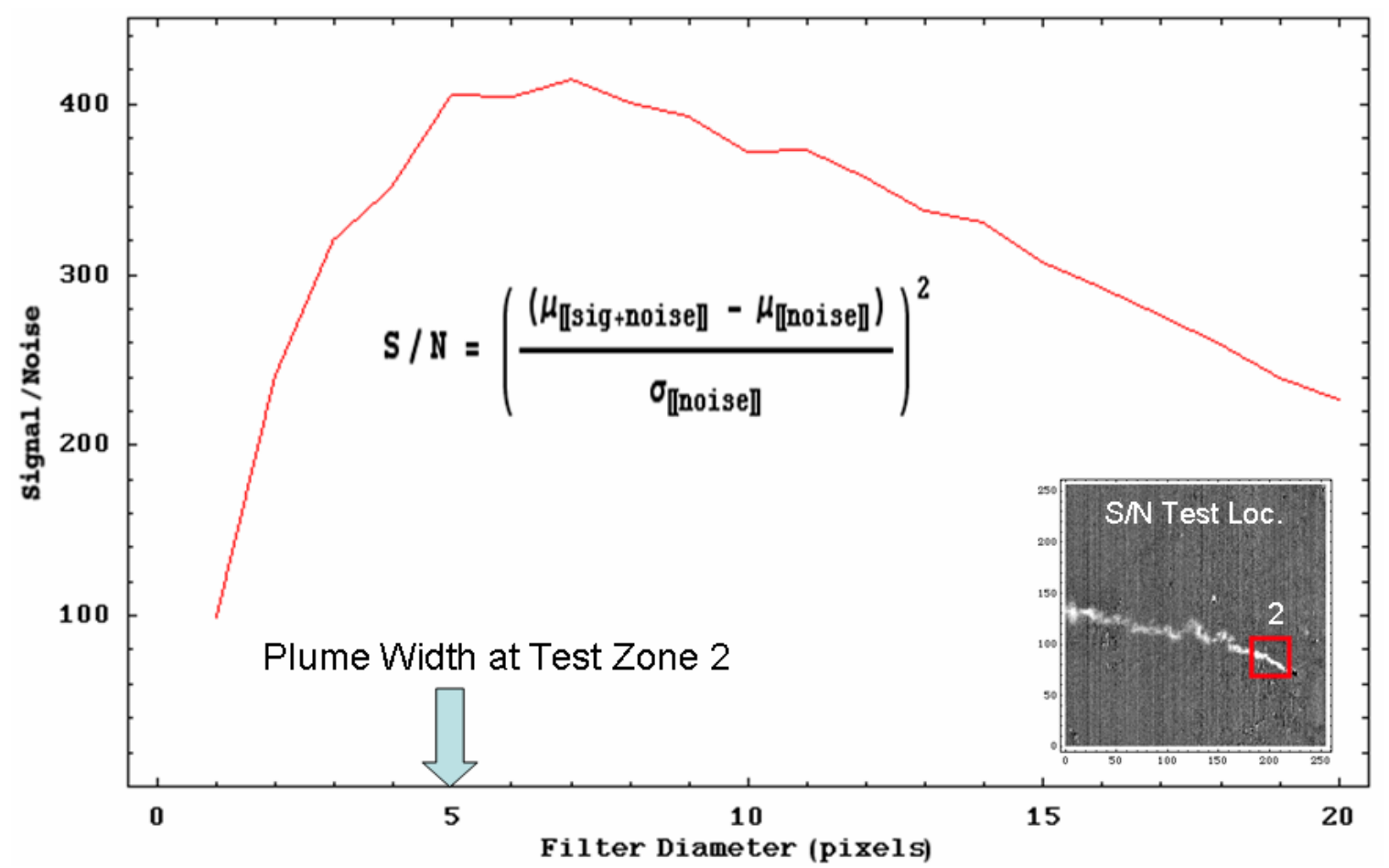

Fig. 16. S/N at test case 2 as a function of spatial filter diameter. In this case $S / N$ peaks near 5 pixels, the plume width in the test zone.

\section{Hyperspectral datacube analyses - Urban scenario}

For further analyses of the 3DFFTWF we compared the results with other techniques on 3 chemical releases in an urban scenario (Fig. 17). The results from PNNL's WMF, 3DFFTMF, and HIP were similar as shown in Fig. 18. The masks for are created from a threshold of 2.3 standard deviations above the mean and also are examples of typical masks for these datasets. To statistically compare the methods the original data would need to be subjected to the same whitening method.

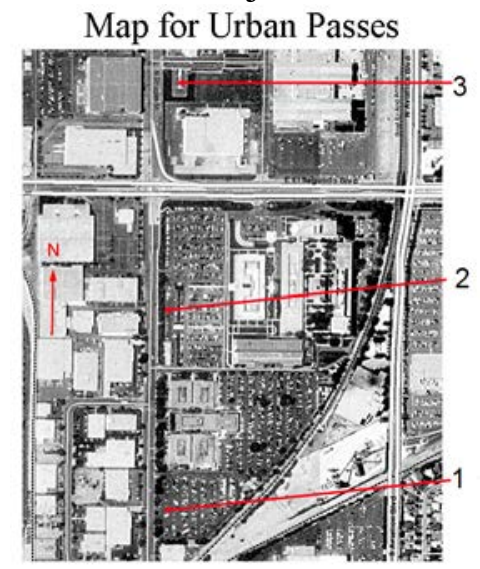

Figure 17. A display of the plume locations relative to types of clutter. 


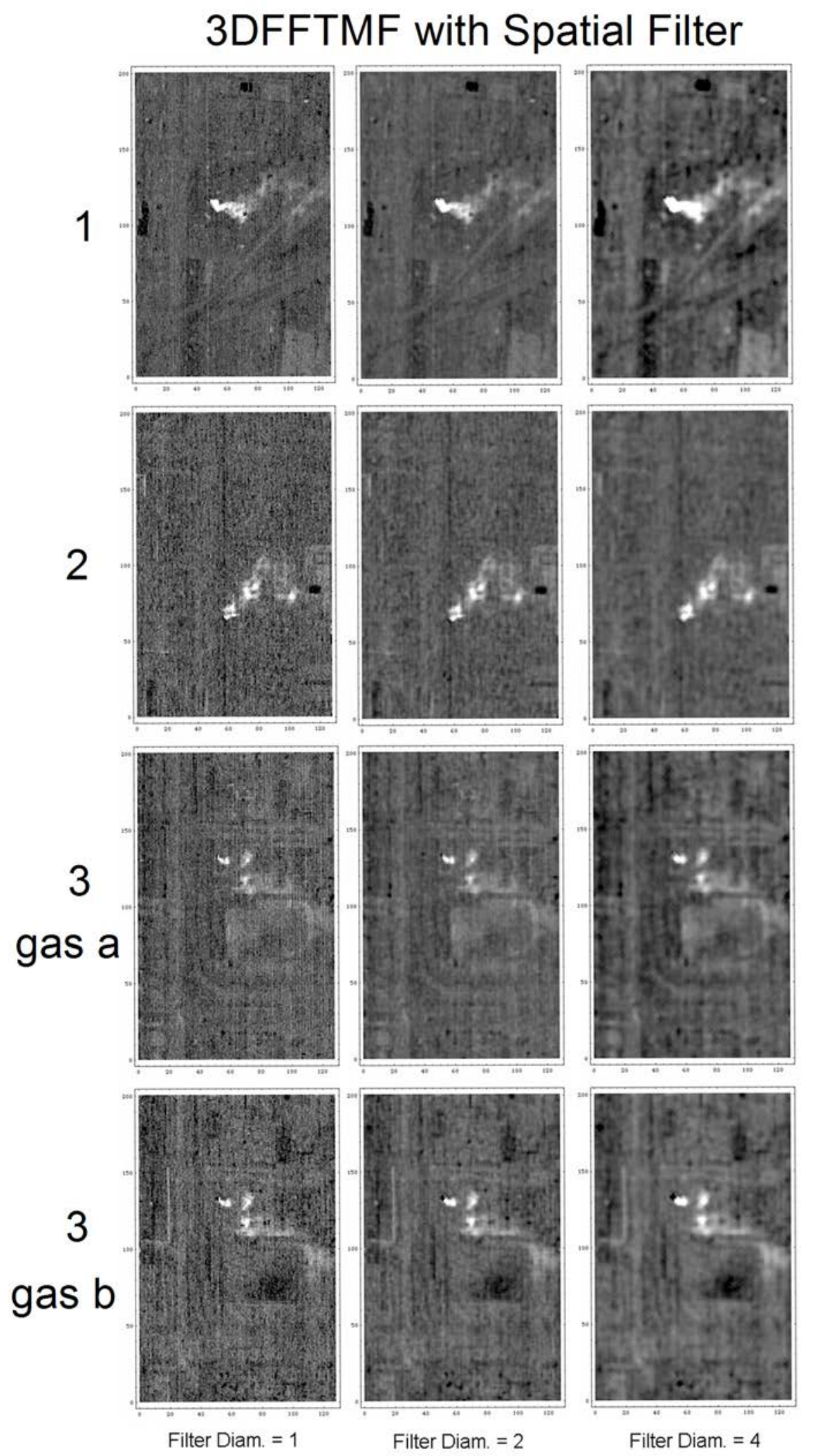

Fig. 18. 3DFFTWF output from urban hyperspectral datacubes. Successively larger low pass filters were applied within Fourier space to reduce the high frequency noise and, 
thus, increase the signal visibility. S/N improvement depends on the relationship between the size of the filter and the size (diameter) of the plume. The spatial filtering will also reduce the mean of the $S+N$ area when The filter diameter approaches the size of the high concentration portion of the plume. In the polecat data the sharp peak of the plume will be Rounded off some with a filter size of 5 pixels which will reduce the mean Some even without the saturation problem. I would guess that for that size Filter the reduction in the noise sigma would be the stronger factor in the $\mathrm{S} / \mathrm{N}$. A plume with a small sharp spike would be affected the most.

Figure 19 displays results from the multiple whitened matched filter techniques, WMF, 3DFFTWF, and HIP with default parameters. The results are similar, without gross disagreements between the different versions of the whitened matched filters. The Mask column was based on the PNNL WMF and is the result of using a threshold on the image of 2.3 standard deviations added to the mean of the pixel intensities combined with selective removal of a few outliers. For true quantitative comparisons the same whitening technique needs to be applied to the original data for creating the comparison image.

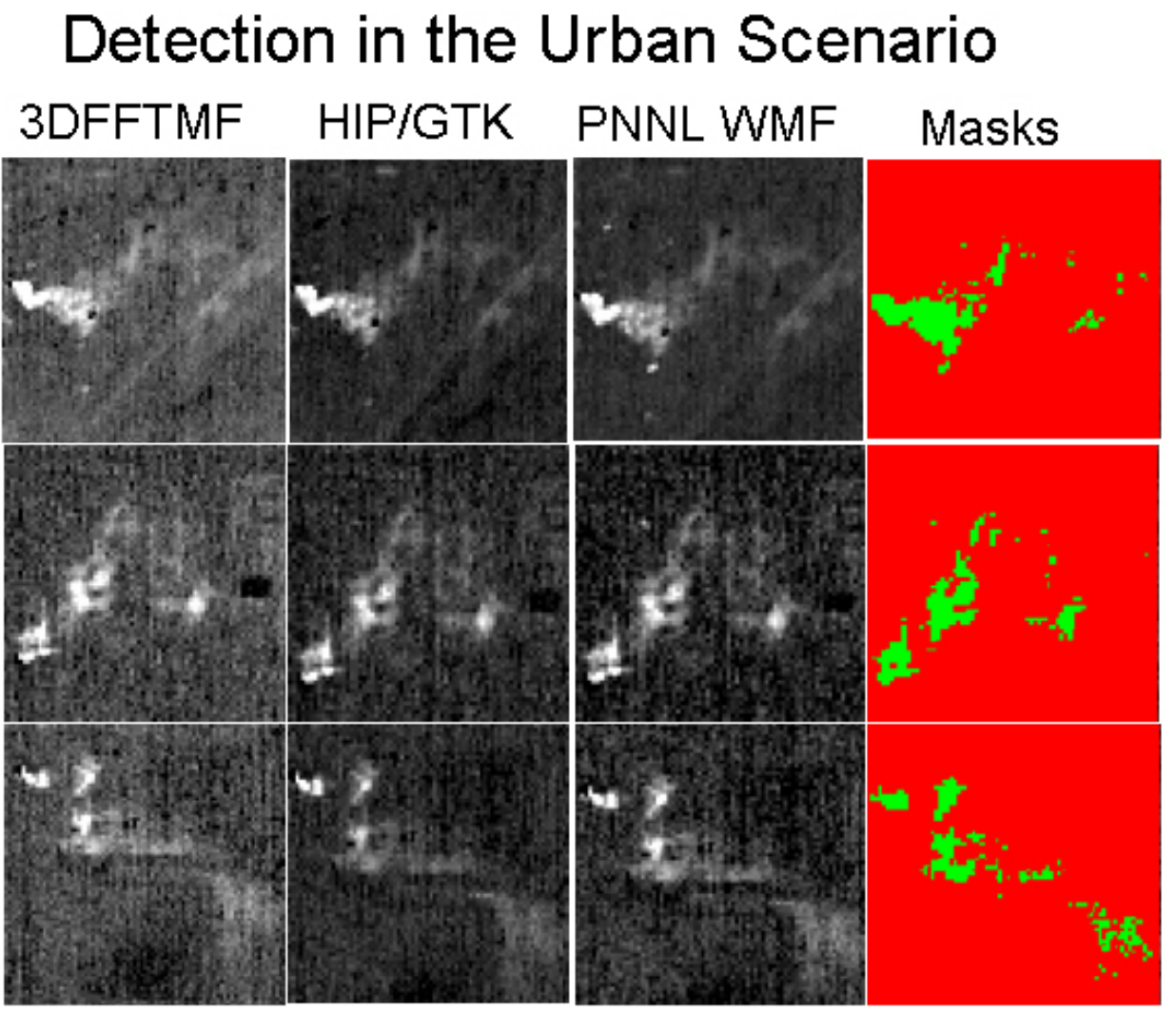

Fig. 19. A comparison of the results from PNNL's 2 whitened match filter programs and HIP on three datacubes from the urban scenario. The masks were created from the PNNL WMF output images as examples of typical masks for these data sets. 


\section{Notes on 3DFFTWF alignments}

Want to match the results from possible paths that are mathematically the same. The first output band of the 3DFFT should contain the relevant results. The other bands are superfluous, not the goal of the comparison with the spectral bands of the object of interest. An assumption that the garbage bands would look the same if done either way. Correlated spatially with plume shifted off, good reproduction of background is output, in later bands the clutter is not removed, they are not just noise representations. Only when properly synchronized with the spectrum is the remainder noise?? Rotation experiment will enable checking whether all be ??? Spatial side whitened cube and do the same test. The effect should be the order of multiplication. Intentionally rotate the filter and correlate that with the whitened cube. This will make sure that all possible combination patterns for the calculations are explored and effects noted.

\section{Future Work}

Detailed issues of the FFT domain whitening process are being analyzed in the Fourier representation using a refined form of the smoothing functions for transforming the periodogram into a whitening spectrum. Comparisons with inverse Covariance whitening are similar. Whitening in both domains is now to be compared in a similar manner with the added target and WMF detections. Future work will include developing Fourier-domain specific whitening techniques, and compare the results to the performance of the standard WMF approach.

\section{Conclusions}

-The 3DFFTMF output based on a spectral signature is similar to the output from standard WMFs.

-Post analysis spatial filters of the approximate width of the item of interest can significantly improve the $\mathrm{S} / \mathrm{N}$.

-Calculation in fourier domain permits connurrent spatial and spectral filtering -Due to the well-developed techniques to calculate FFT and inverse FFT the 3DFFTWF can have an analytical advantage over the standard WMFs.

\section{References}

Bodrero, D. R., J. G. Yale, R. E. Davis, and J. M. Rollins. 1999. A Temporally Rendered Automatic Cloud Extraction (TRACE) System. http://www.dtc.army.mil/tts/1999/proceed/bodrer99/index.html

Lundberg, M. and I.Y.H. Gu. 2000. A 3-D matched filter for detection of land mines using spatio-temporal thermal modeling. Spie Proceedings: Detection and Remediation Technologies for Mines and Minelike Targets Spie Proceedings: Detection and Remediation Technologies for Mines and Minelike Targets V. http://publications.lib.chalmers.se/records/full_record/14866.html or http://www.s2.chalmers.se/research/signal/projects/projectdev_personal.php?user=mlg\&d $\mathrm{b}=$ author=lundberg 
Medvick, P. A., L. L. Nuffer, H. P. Foote, M. A. Lind. 2007. Adaptive Whitening for Rapid Processing of Hyperspectral Images. PNNL Report: PNNL-16658.

Oppenheim, A.V. and J. S. Lim. 1981. The Importance of Phase in Signals.

Proceedings of the ICC, Vol. 69, No. 5, pp. 529-541.

http://ieeexplore.ieee.org/iel5/5/31301/01456290.pdf

Young, S. J., 2002. Detection and Quantification of Gases in Industrial-Stack Plumes Using Thermal-Infrared Hyperspectral Imaging. Aerospace Report No. ATR2002(8407)-1. 